\title{
A Multi-level Analysis of the Relationship between Instruc- tional Practices and Retention in Computer Science
}

\author{
Markeya S. Peteranetz \\ College of Engineering \\ University of Nebraska-Lincoln \\ Lincoln, NE, USA \\ peteranetz@unl.edu
}

\author{
Leen-Kiat Soh \\ Dept. of Computer Science \& Engineering \\ University of Nebraska-Lincoln \\ Lincoln, NE, USA \\ lksoh@cse.unl.edu
}

\begin{abstract}
Increasing retention in computer science (CS) courses is a goal of many CS departments. A key step to increasing retention is to understand the factors that impact the likelihood students will continue to enroll in CS courses. Prior research on retention in CS has mostly examined factors such as prior exposure to programming and students' personality characteristics, which are outside the control of undergraduate instructors. This study focuses on factors within the control of instructors, namely, instructional practices that directly impact students' classroom experiences. Participants were recruited from 25 sections of 14 different courses over 4 semesters. A multi-level model tested the effects of individual and class-average perceptions of cooperative learning and teacher directedness on the probability of subsequent enrollment in a CS course, while controlling for students' mastery of CS concepts and status as a CS major. Results indicated that students' individual perceptions of instructional practices were not associated with retention, but the average rating of cooperative learning within a course section was negatively associated with retention. Consistent with prior research, greater mastery of CS concepts and considering or having declared a CS major were associated with a higher probability of taking a future CS courses. Implications for findings are discussed.
\end{abstract}

\section{CCS CONCEPTS}

Social and professional topics Computer science education

\section{KEYWORDS}

Computer science education; Retention; Multi-level models; Cooperative learning; Student-centered classrooms

Permission to make digital or hard copies of all or part of this work for personal or classroom use is granted without fee provided that copies are not made or distributed for profit or commercial advantage and that copies bear this notice and the full citation on the first page. Copyrights for components of this work owned by others than the author(s) must be honored. Abstracting with credit is permitted. To copy otherwise, or republish, to post on servers or to redistribute to lists, requires prior specific permission and/or a fee. Request permissions from Permissions@acm.org. SIGCSE '20, March 11-14, 2020, Portland, OR, USA

(C) 2020 Copyright is held by the owner/authors. Publication rights licensed to ACM. ACM 978-1-4503-6793-6/20/03...\$15.00

https://doi.org/10.1145/3328778.3366812

\begin{abstract}
ACM Reference format:
Markeya S. Peteranetz and Leen-Kiat Soh. 2020. A multi-level analysis of the relationship between instructional practices and retention in computer science. In Proceedings of ACM SIGCSE conference (SIGCSE'20), March 1114, 2020, Portland, OR, USA. ACM, New York, NY, USA, 7 pages. https://doi.org/10.1145/3328778.3366812.
\end{abstract}

\section{INTRODUCTION}

In recent years in the United States, there has been an influx of interest and energy in growing computer science (CS) education across all educational levels. From the CSforAll consortium in the United States to the increase in European countries that have added coding to the elementary and secondary school curriculum [6], there is growing consensus that knowledge of CS concepts, computational thinking skills, and familiarity with coding will play an important role in future workforce readiness. In order for the United States to meet future workforce demands for CS professionals [15], it is important for tertiary CS programs to attract and retain students. However, at the collegiate level, college STEM disciplines have historically seen large proportions of students not completing the degree they initially pursue [26, 30], and $\mathrm{CS}$ is no exception [9].

A history of low retention and degree completion rates in CS $[13,14]$ have prompted research into factors contributing to retention because understanding these factors can help educators develop strategies to attract and retain students in CS programs. For example, researchers have found prior exposure to CS and programming [13], personality characteristics [1], motivation for a given course [23], the learning environment [9], and achievement in introductory CS courses [13] are factors that predict students' continued enrollment in CS courses. In addition to these factors, students' experiences in classes within a certain discipline can impact whether they keep taking classes [2, 5, 31].

To date, we are unaware of any research on the impact of CS students' perceptions of instructional practices on their retention in CS courses. The present study is intended to help fill this gap in the literature. As instructors, programs, and universities seek to improve retention rates, it is especially important to understand factors related to retention that are within the control of educators. Many aspects of instruction are under the complete or 
near complete control of the teacher, making instructional practices a potentially fruitful area for interventions that can impact retention.

The purpose of this study was to examine how retention in CS courses is impacted by (a) students' perceptions of an information-transmission orientation to teaching (later called teacher directedness, TD) and (b) the perceptions of peer cooperation and interaction within a course section (later called cooperative learning, CL). The research question guiding this study was, do students' perceptions of instructional practices impact the likelihood they will enroll in at least one CS course the following semester? In the next section, we review research related to this research question. Then, we describe the study that was conducted, followed by results of the study, a discussion of the findings and their implications, and finally, we present concluding remarks.

In addition to contributing to the CS education literature by addressing the main research question, this study also contributes to the field by demonstrating an application of a multi-level logistic regression model within a CS education context. Multi-level models (MLMs) are used within education research to account for "clustering" within data, which naturally occurs in samples that consist of students who are clustered within a set of courses, a set of schools, or both. In this study, students were recruited from 25 different course sections over 4 semesters. Failing to account for this type of clustering within a dataset biases statistical estimates because individuals within a cluster tend to be more similar to each other than to individuals from different clusters because of the shared influence of the environment of their cluster (in this case, being in the same course section). As CS education research continues to grow and studies continue to draw larger samples from multiple courses and institutions, it will become more and more necessary for scholars to be familiar with MLMs and their applications in order to account for clustering biases in their studies.

\section{RELATED RESEARCH}

\subsection{Perceptions of the Classroom}

Students' perceptions of the classroom influence their behavior related to that course. Importantly, students' perceptions can differ markedly from teachers' beliefs about students' perceptions of their course. For example, this difference is evident to every instructor who has been shocked by end-of-term student evaluations. Because instructors are not always able to objectively judge the climate of their classroom or the way their instruction is perceived, it is important to consider the role of students' perceptions. Researchers in a variety of disciplines have examined the impacts the perceived classroom environment has on multiple outcomes. For example, [25] studied the relationship between college students' perception of the classroom environment, cheating, and justifications for cheating. Across a range of disciplines, aspects of students' classroom perceptions were associated with cheating in that course. Specifically, students who perceived the course as being better organized and were more satisfied with their experiences in the course were less likely to cheat. Classroom perceptions were also related to justifications for cheating, whether or not the student had cheated (or admitted to cheating).
Students reported greater justifications for cheating when (a) they felt less of a personal connection to their instructor, (b) they reported less engagement during classes, (c) felt less collegiality toward classmates, (d) were less satisfied with the course, (e) perceived classes as less organized, and (f) felt less autonomy and control over their work in the course.

In [3], undergraduate chemistry students' perceptions of the relative emphasis of learning and assessment outcomes (e.g., grades), cognitive engagingness of lectures, and the harshness of grading practices were related to the types of achievement goals they set for the course, which in turn impacted their achievement and motivation for the course. Students who perceived the instructor as being focused on grades, rather than learning, were less likely to set goals to master course content (mastery goals) and were more likely to set goals related to their final grade and others' judgments of their competence (performance goals). Students who perceived grading practices as harsh were also less likely to set mastery goals and were more likely to focus on not failing (performance-avoidance, as opposed to goals to work toward a desirable grade, performance-approach). Finally, students who judged lectures as engaging were more likely to set mastery goals and were less likely to set performance-avoidance goals. Course perceptions had an indirect impact on motivation and course grades, with mastery goals positively related to both outcomes, performance-avoidance goals negatively related to both, and performance-approach goals positively related to grades.

Finally, [32] studied the relationships between marketing students' perceptions of the classroom "climate" and self-regulated cognition, motivation, and behavior. Students' perceptions of the classroom climate were predictive of their achievement goal orientation, perceived competence, and perceived autonomy in the course. Additionally, perceptions of the climate related to grades and performance evaluation in the course were predictive of students' use of planning strategies when studying for the course.

\subsection{Interventions Targeting Retention}

There are numerous examples of interventions aimed at increasing retention in science, technology, engineering, and mathematics (STEM) disciplines being implemented, evaluated, and reported through peer-reviewed publication. For example, Wilson et al. [31] described a program implemented at the Howard Hughes Medical Institute at Louisiana State University (LSUHHMI). The LSU-HHMI Professors Program integrated mentoring, undergraduate research experiences, and supplemental academic and professional development opportunities through a multi-tiered mentoring model. Students in the mentoring program had higher six-year graduation rates than LSU STEM students not in the program and STEM students across the US who enrolled in a STEM program in the same year.

As a second example, Dagley et al. [5] described a STEM learning community that increased undergraduate students' retention. The two-year EXCEL program at the University of Central Florida incorporates residential, (an on-campus learning community) social (STEM-oriented social events), and curricular (undergraduate research experiences and cohort-based math courses) components. Analysis of student retention over several years indicated 
that students in the EXCEL program were more likely to still be enrolled in their STEM major one year after matriculation and were more likely to graduate than comparable STEM students who did not participate in the EXCEL program during the same time period.

Though programs like those described in [31] and [5] have been shown to be effective, they are comprehensive, resource-intensive programs that can only be implemented if key personnel at multiple levels of an institution agree to devote the resources to implementing such a program. When budgets are tight or being cut or other barriers to large-scale change exist, smaller interventions that can be implemented by one or a few committed individuals are a more viable option. In today's climate, academic units are asked to do more for an increasing number of students with fewer financial resources. As a result, it is increasingly important for efforts to increase retention to be less resource-intensive than comprehensive programs like the ones described above. Program- or department-level changes and classroom-level instructional choices are two possibilities for increasing retention while placing a minimal demand on resources.

A few program-level changes and classroom-level interventions have been shown to increase CS majors' retention in CS. For example, Ott et al. [21] reported a program-level change that impacted student retention. At the studied institution, two versions of introductory CS were offered: one for students with programming experience and another for students with no prior programming experience. For several years, students could opt-in to the course for students with programming experience, but relatively few students with programming experience did. . When students were required to take a placement exam, more students enrolled in the course that was appropriate for their level of prior experience, and the change in placement procedures increased the likelihood that students would continue taking CS courses. In a classroom-level intervention reported by Carver et al. [2], pair programming was used during an introductory course's laboratory exercises. Pair programming is a collaborative approach to programming activities that involves pairs of students who take turns working in two roles, the programmer or "driver" and the debugger or "navigator" [28]. First-year students in introductory programming courses that used pair programming were more likely to persist in their computing major than students in the same courses that did not include pair programming activities [2]. In both of these examples, an intervention heavily tied to the CS curriculum was successful in increasing retention among students with a computing major. In contrast, our interest lies with determining whether aspects of the instructional practices impact whether students take additional CS courses.

Classroom-level changes that might impact student retention, such as choices related to instructional practices, are relatively low-cost and simple to implement, as compared to the large-scale programs outlined by [5, 31]. For example, the model put forth by Graham et al. [10] identified instruction that incorporated active learning as a contributor to students' persistence in STEM programs. Active-learning instruction, which can include cooperative learning $(\mathrm{CL})$, is a relatively low-cost way to potentially im- prove the learning experience of all students, majors and non-majors alike. It is possible that multiple aspects of instruction are related to the likelihood that students will continue taking CS courses. Understanding how instructional practices are associated with retention can provide a basis for implementing classroom-level interventions for increasing retention in CS.

\section{METHOD}

The study was approved by the university IRB and students participated voluntarily in data collection. Data used in this study were collected as part of a larger NSF-funded study of computational thinking in undergraduate CS. Only the data collection and materials that pertain to the present study are described here.

\subsection{Participants and Procedure}

Participants in the study were undergraduate students enrolled in $\mathrm{CS}$ courses at a large, public, Midwestern university $(\mathrm{N}=607 ; 502$ male, 105 female). The sample contained students at all academic levels (213 freshmen, 206 sophomores, 129 juniors, 59 seniors) and were recruited from five 100-level courses $(n=420)$, one 200-level course $(n=64)$, three 300 -level courses $(n=82)$, and five 400 -level courses $(n=41)$ over four semesters. The number of unique sections (i.e., the number of clusters in the analysis) was 25 .

Survey-based data collection took place three times during the semester: the first week, around the eighth week (depending on the timing of each course's events), and the week prior to final exams. The surveys contained instruments that assessed aspects of students' motivation, self-regulation, course-related affect, studying behaviors, and perceptions of classroom instruction as well as demographic information. The end-of-semester survey also included a test of core CS concepts (described in Section 3.2).

Upon consenting to participate in the study, participants had the option of granting the researchers access to their future course enrollment. Enrollment data were obtained from the university after the open drop/add period for that semester had ended. Students who did not enroll in any classes during the semester following their participation in the study were not included in the analysis because of the multiple reasons students might not enroll in classes (e.g., graduation, transferring, dropping out of school). As a result, the comparison is of students who enrolled in a CS course and students who were enrolled at the university, but did not enroll in a CS course during that semester. Enrollment data were coded dichotomously and indicated whether students did (enrollment $=1)$ or did not (enrollment $=0$ ) enroll in a CS course the following semester. Instances of students re-enrolling in the course from which they were recruited were not counted: students retaking a course had to be enrolled in at least one additional course to be counted as enrolling in CS during that semester. In the sample, $53.4 \%$ of participants $(n=356)$ enrolled in a CS course the following semester.

\subsection{Predictor Variables}

Student perceptions of instructional practices were measured by the Student Perceptions of Classroom Knowledge Building (SPOCK) scale. The SPOCK is a course-specific instrument that 
measures students' self-regulation and use of learning strategies, question-asking behaviors, and perceptions of the classroom environment, including $\mathrm{CL}$ and TD. A shortened version of the SPOCK that has been used in other studies [7, 27] was used here, and only the items relating to the classroom environment (Cooperative Learning and Teacher Directedness subscales) were included in the analysis. The shortened version contains 27 items that make up the same subscales as the full version. Items in the SPOCK are paired with a 5-point response scale that ranges from "almost never" to "almost always". Each response category has a brief description (e.g., Often, occurred frequently: occurred about $3 / 4$ of the time) to assist respondents in interpreting the labels.

The Cooperative Learning subscale contained 3 items ("In this class, my classmates and I actively worked together to help each other understand the material." "In this class, my classmates and I actively worked together to complete assignments." "When I did my work in this class, I got helpful comments about my work from other students."). For this sample coefficient alpha was .82. The Teacher Directedness subscale contained 3 items ("In this class, the instructor focused on getting us to learn the right answers to questions." "In this class, the instructor told us what the important information was." "In this class, the instructor gave us specific instructions on what we were to do."). For this sample coefficient alpha was .78.

Scores on the Nebraska Assessment of Computing Knowledge (NACK) were used to control for the relationship between students' mastery of CS content and retention. The NACK is a 13item multiple-choice test of core CS concepts. It served as a measure of CS mastery that was uniform across courses and instructors. The test has been used in prior studies and information on its development is available in [20,27]. Coefficient alpha for this sample was .78.

Participants' self-report of their status as a declared CS major was used to control for the relationship between one's major and retention. For the question, "Are you considering a major or minor in Computer Science/Computer Engineering?" the response options were "Yes," "No," and "I am already majoring/minoring in Computer Science/Computer Engineering. Because declared or intended major/minor is one of the strongest influences on the classes one takes, we included major status as a control in our model. The three options were dummy coded into two binary variables: one for "considering" status (considering = 1 , else $=0$ ), one for "majoring" status (major $=1$, else $=0$ ), leaving the intercept as those not considering or majoring in CS or computer engineering.

\subsection{Multi-level Models (MLMs)}

In education research, samples are often drawn from naturally clustered systems, such as students nested within courses that are nested within schools. Clustering introduces bias into statistical estimates because of the probability that individuals within the same cluster are more similar (in terms of measured and unmeasured variables) than individuals from different clusters. Multilevel models $[8,12]$ make it possible to account for multiple levels of clustering within data, and can be used with a range of classic single-level statistical approaches including regression, randomcoefficient models, and structural equation modeling (SEM).
When generating a MLM, one specifies relationships among variables at the within- or micro- level (also, Level 1) and the between-or macro-level(s) (also, Level 2, Level 3, etc.), and possibly, between levels. In the present study students were nested within course sections, making Level 1 the student level and Level 2 the section level (there were not enough unique courses to model a third level for course). The Level 1 portion of our model specifies relationships between Level 1 predictor variables-perceptions of instructional practices, major status, and achievement-and Level 1 outcomes-enrollment in a CS course the following semester. The Level 2 portion of our model specifies relationships between Level 2 predictors-the section mean of perceived instructional practices-and Level 2 outcomes-the section mean for enrollment in a CS course the following semester. A logistic regression model was used because enrollment was coded as a dichotomous variable. Figure 1 shows a visual depiction of the model.

A statistic of interest for MLMs is the intra-class correlation (ICC). The ICC indexes the amount of variability in the outcome (here, retention) that is accounted for by the clustering included in the model (here, course sections). If there are large differences in the outcome variable between groups (course sections) but little difference within each group, the ICC will be close to 1 . Conversely, if there are large differences within groups and group means are nearly identical, the ICC will be close to 0 . In this study, a high ICC would indicate most students in some course sections took subsequent CS courses and most students in other courses did not, and a low ICC would indicate the rate of retention was similar across all course sections.

Other statistics of interest for the model used in this study are (1) $R^{2}$, (2) regression coefficients, and (3) odds ratios. In logistic regression, the interpretation of $R^{2}$ is similar to that of regression with continuous outcomes. Logistic regression coefficients estimate the change in the natural log of the odds that the outcome will occur (here, enrollment $=1$ ) that is associated with every 1 unit increase in the predictor variable, and thus, are not intuitively interpretable. The odds ratio, however, indicates the change in the odds of the outcome occurring that is associated with a 1-unit increase in the predictor variable, and is more readily understandable. For example, an odds ratio of 2.0 for majoring or not majoring in CS in this study would indicate that the students already majoring in CS $(x=1)$ were twice as likely to take a CS course as students not already majoring in CS $(x=0)$.

Finally, the fit statistics used for model comparison were the Akaike Information Criterion (AIC) and the Bayesian Information Criterion (BIC). AIC and BIC can be used to compare the fit of related models. They take into account model fit (in terms of log likelihood) and model complexity (in terms of the number of parameters estimated), with BIC giving a steeper penalty for additional parameters. For both statistics, smaller values indicate a better fitting model, but because they are heavily influenced by the nature of the tested model, there are no conventions or cutoffs for interpreting them like there are for some other fit statistics.

The analysis for this study was conducted in Mplus V. 8.1 [18] using the analysis command "TYPE = TWOLEVEL," indicating a two-level clustering structure and the inclusion of randomly varying intercepts for each cluster (i.e., a separate cluster mean was 


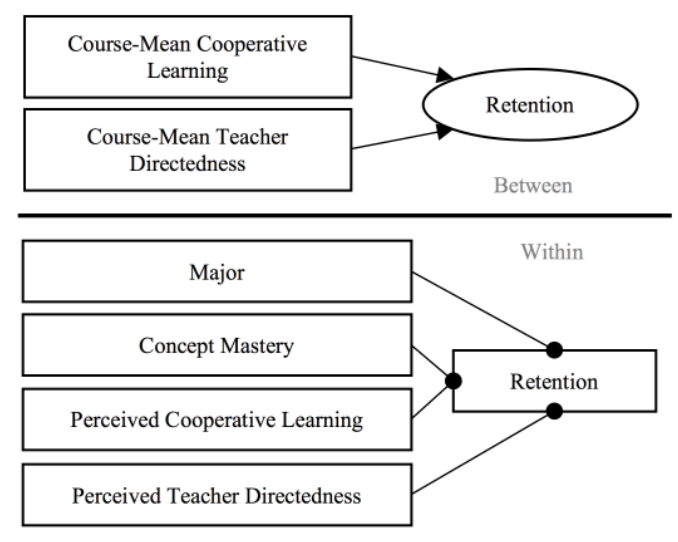

Figure 1: Diagram of the Tested Multi-level Model.

estimated for each cluster). The cluster variable indicated the unique course section from which participants were sampled. Including a random intercept for course section is a way to account for uniqueness associated with a given cluster, including the instructor, the course level, and the shared environment of the course section. Student ratings of CL and TD included in Level 1 were centered within-cluster so that estimated parameters would reflect a students' perception of the environment, relative to his or her classmates. This method of centering also makes it possible to separate the individual-level effects from the class-level effects [16]. The indicators of CL and TD included in Level 2 were cluster means, therefore capturing the relationship between average ratings of CL and TD and proportion of students in a cluster that enrolled in a CS course the following semester. Assuming that the group average ratings of instructional practices approximates actual instructional practices, the inclusion of centered-within-cluster parameters and group mean parameters allowed us to model the effect of the individual's perception on retention as well as the effect of the actual instructional environment.

When using aggregations of individual ratings as an indicator of a classroom-level variable, the reliability of the classroom-level variable must be checked [16]. This is done by calculating the ICC for the variable and then using the Spearman-Brown formula to adjust the ICC according to the average number of units (here, students) per cluster (Eq. 2 in [16]). The estimate of reliability of the aggregate variable increases as the cluster size increases, similar to the expected increase in the reliability of test associated with increasing the number of items on the test.

\section{RESULTS}

\subsection{Baseline Model}

First, we tested a baseline model to determine the extent to which participants' section predicted their enrollment. The ICC for the empty model was 0.734 , indicating the majority of variability in enrollment was accounted for by the course section in which

Table 1: Model Statistics

\begin{tabular}{lccc}
\hline Model & AIC & BIC & ICC \\
\hline Empty & 504.77 & 513.77 & 0.743 \\
Full & 363.33 & 403.13 & 0.476 \\
\hline
\end{tabular}

Table 2: Logistic Regression Statistics for the Full Model

\begin{tabular}{lccccc}
\hline & $B$ & S.E. & Wald & $p$ & $\begin{array}{c}\text { Odds } \\
\text { ratio }\end{array}$ \\
\cline { 2 - 6 } Level 1 & & & & & \\
CL $^{*}$ & 0.17 & 0.15 & 1.14 & .256 & 1.18 \\
TD $^{*}$ & -0.18 & 0.20 & -0.89 & .374 & 0.84 \\
CS Knowledge Test & 0.14 & 0.06 & 2.47 & .013 & 1.15 \\
Considering Major & 3.10 & 0.35 & 8.80 & $<.001$ & 22.11 \\
Already a Major & 3.81 & 0.66 & 5.74 & $<.001$ & 44.98 \\
Level 2 & & & & & \\
CL & -1.33 & 0.66 & -2.01 & .044 & -- \\
TD & 0.12 & 0.90 & 0.13 & .893 & -- \\
\hline
\end{tabular}

Note. $B$ = regression coefficients, S.E. = standard error, $\mathrm{CL}=$ cooperative learning, TD $=$ teacher directedness, ${ }^{*}$ centered-in-cluster, ${ }^{* *}$ cluster mean.

participants were enrolled. ICCs and fit statistics for both models are shown in Table 1. The high ICC was unsurprising, as students in advanced courses were likely CS majors who would take additional CS courses and some of the introductory sections at this university were mostly taken by non-CS majors. Because our hypothesized model included Level 2 predictors (i.e., predictors related to characteristics of the different course sections), we expected the ICC to be lower once the predictors were added.

\subsection{Reliability of Aggregate Variables}

The reliability of the aggregate classroom perception variables was then estimated. This was done by following the procedure described in Section 3.3. For Cooperative Learning, ICC $=0.332$, and for Teacher Directedness, ICC $=0.203$. The average number of students per cluster was 26.68 . As a result, the reliability estimates for the class means were $\mathrm{CL}=0.930$ and $\mathrm{TD}=0.872$.

\subsection{Hypothesized Model}

The "full" hypothesized model that included all the predictor variables was then tested. Model fit statistics indicate the full model better approximated the data than the empty model. As expected, the ICC for the full model was lower, ICC $=0.476$, than for the empty model, indicating the set of predictors partially explained the differences between clusters. The predictors and their statistics are shown in Table 2.

- As expected, the control variables were significant. Majors were 45 times more likely and those considering a CS major were 22 times more likely to enroll in a CS course than nonconsidering non-majors. Higher mastery of course content was associated with a slightly higher likelihood of taking additional CS courses.

- Level 1 perceptions of CL and TD were not significant.

- Interestingly, a higher cluster mean rating of CL was associated with a lower likelihood of taking additional CS courses.

\section{DISCUSSION AND IMPLICATIONS}

This paper demonstrates an application of a MLM in CS education research. Because of the naturally occurring nesting in education settings, MLMs are needed when samples are drawn from multiple courses or institutions. As the CS education field continues to grow and conduct larger studies, it will be necessary for researchers to become familiar with and be able to implement MLMs. 
Of central importance to this paper, students' individual perceptions of instructional practices were not associated with retention. A student who perceives the classroom as using more peer cooperation or as being more teacher-directed is no more or less likely to take additional CS courses than a student who perceives that same classroom environment as less so. A second central finding of this paper is that the aggregate rating of $C L$ in a course section was associated with a lower retention rate. It is possible that this unexpected relationship is due to a greater emphasis on using CL in introductory courses, which contain higher numbers of non-major students who are unlikely to take CS beyond that level.

Reviewing the course section means for CL revealed, not that CS1 courses were consistently higher in using CL, but that CS1 course sections tended to be more similar in their aggregate ratings of CL (Ms from 2.43 to 3.56) than upper-level courses (Ms from 1.57 to 3.86 ). At this time, it is unknown how this variability in the extent to which CL is used in upper-level courses might impact students' experiences and satisfaction with their course, but future research should explore this topic.

The measures of perceived instructional practices included in this study were targeted at general instructional practices, and did not include CS-specific practices that are prominent in the literature. Future research should examine the impact of more specific instructional practices on retention, such as active learning [17], pair programming [24, 29], game-based learning [22], contextbased instruction [4], and the use of multimedia [11]. A final point of importance is that the general instructional practices examined here were not predictive of retention in CS, but concept mastery was, underscoring the importance of using high-quality, evidence-based teaching practices that increase the likelihood that students will master CS concepts and skills.

Unsurprisingly, the strongest predictor of students' continued enrollment in CS courses was their status as a major or consideration of a major in CS. Because students' majors have an exceptionally large influence on the courses they take, it is critical to take major into account when studying retention in a specific discipline. The sample in this study included students in introductory CS (CS1) courses that were required for their non-CS major, with engineering students comprising a substantial portion of students in the CS1 course sections. It also included CS1 sections for CS majors and upper-level CS courses that were overwhelmingly taken by CS majors. Even after accounting for the different sections students were in (by using a MLM), students' individual status as a major, non-major, or considering a major strongly predicted whether they would take an additional CS course. It should be noted, however, that the modes tested in this study did not include random slopes, and thus specified that the relationship between the instructional practices variables and retention is the same for all of the courses. Future research (with a larger sample of course sections) should consider the possibility of differences in this relationship by including random slope parameters.

Students scoring higher on the test of core CS concepts were also more likely to continue taking CS courses. However, the causality of this relationship cannot be determined from this study: it is possible that students who have greater mastery of the course perceive that mastery as a cue they should continue in the discipline, but it is also possible that students who have decided to take multiple CS courses are more likely to master the content. In all likelihood, both explanations are at least partially true.

Given the above observations, we propose that the reason is that students' decisions to take additional courses in a discipline is based on their major and future career plans, which has been shown to be related to retention in CS [19], and is not influenced much by the instructional practices used in any one course. It should be noted that there is abundant research indicating instructional practices can influence students' learning: this explanation does not dispute that literature, but rather suggests that the relationship of instructional practices with learning is separate from the relationship with retention.

\section{CONCLUSION}

As CS educators continue to be concerned with increasing retention rates of students in undergraduate programs, it is important for the community to better understand the factors that contribute to success and retention in CS. This study examined how four factors, (1) students' perceptions of instructional practices, (2) classlevel indicators of instructional practices, (3) students' status as a CS major, and (4) students' mastery of course content, are associated with the likelihood students will continue enrolling in CS courses. Through multi-level modeling, these relationships were tested simultaneously across a large number of course sections that included courses from CS1 to senior-level courses. Findings from this study indicate that after controlling for students' individual plans related to majoring in CS and their mastery of CS concepts, the general instructional practices used in the course section do not predict retention. Additional research on other CS-specific instructional practices might help CS educators identify those practices that can encourage students to keep taking CS courses.

One of the contributions from this study is the use of multilevel modeling. Without multi-level modeling, it would not be possible to analyze the relationships of course-level variables across a set of courses, and person-level estimates would not be accurate. Had only the person-level variables been used in this study, the course-level effect of CL would not have been detected.

In addition to investigating the impact of other instructional practices on retention, future research should further study the role of content mastery and students' majors on retention. With content mastery, more closely examining the aspects of core CS content that predicts retention and success in later classes could provide insight into how course topics should be timed, reviewed, and paired with other interventions designed to increase retention. With students' major, considering the possibility of differential impacts of instructional practices on CS majors and non-CS majors could provide insights into which practices are best suited for which groups of students, with respect to retention. If the relationships between instructional practices and retention are different for majors and non-majors, it would suggest that some practices are more suited for CS1 courses and others for later courses. Overall, there is still much to be learned about how instructional practices are related to retention in CS. 


\section{ACKNOWLEDGMENTS}

This work was partially supported by the National Science Foundation (Grants No. 0829647, 1431874, and 1122956).

\section{REFERENCES}

[1] S. Beyer. 2014. Why are women underrepresented in Computer Science? Gender differences in stereotypes, self-efficacy, values, and interests and predictors of future CS course-taking and grades. Computer Science Education, 24(2-3), 153-192.

[2] J. C. Carver, L. Henderson, L. He, J. Hodges, and D. Reese. 2007, July. Increased retention of early computer science and software engineering students using pair programming. In 20th Conference on Software Engineering Education \& Training (CSEET'07) (pp. 115-122). IEEE.

[3] M. A. Church, A. J. Eliot, and S. L. Gabel. 2001. Perceptions of classroom environment, achievement goals, and achievement outcomes. Fournal of educational psychology, 93(1), 43 .

[4] S. Cooper, and S. Cunningham. 2010. Teaching Computer Science in Context, ACM Inroads, 1(1), 5-8.

[5] M. Dagley, M. Georgiopoulos, A. Reece, and C. Young. 2016. Increasing retention and graduation rates through a STEM learning community. Fournal of College Student Retention: Research, Theory \& Practice, 18(2), 167-182.

[6] Euractiv. 2015. Infographic: Coding at school - How do EU countries compare? Retrieved from https://www.euractiv.com/section/digital/infographic/infographic-coding-at-school-how-do-eu-countries-compare/

[7] A. E. Flanigan, M. S. Peteranetz, D. F. Shell, and L.-K. Soh. 2017. Implicit intelligence beliefs of computer science students: Exploring change across the semester. Contemporary Educational Psychology, 48, 179-196.

[8] A. Gelman, and J. Hill. 2006. Data analysis using regression and multilevel/hierarchical models. Cambridge University Press.

[9] M. N. Giannakos, I. O. Pappas, L. Jaccheri, and D. G. Sampson. 2016. Understanding student retention in computer science education: The role of environment, gains, barriers and usefulness. Education and Information Technologies, $1-18$.

[10] M. J. Graham, J. Frederick, A. Byars-Winston, A. B. Hunter, and J. Handelsman. 2013. Increasing persistence of college students in STEM. Science, 341(6153), 1455-1456.

[11] M. Guzdial, and E. Soloway. 2002. Teaching the Nintendo Generation to Program, Communications of the ACM, 45(4):17-21.

[12] R. H. Heck, and S. L. Thomas. 2015. An introduction to multilevel modeling techniques: MLM and SEM approaches using Mplus. Routledge.

[13] S. Katz, D. Allbritton, J. Aronis, C. Wilson, and M. L. Soffa. 2006. Gender, achievement, and persistence in an undergraduate computer science program, The DATA Base for Advances in Information Systems, 37(4), 42-57.

[14] S. Katz, J. Aronis, D. Allbritton, C. Wilson, and M. L. Soffa. 2003. Gender and race in predicting achievement in computer science, IEEE Technology and Society Magazine, 22(3), 20-27.

[15] D. Langdon, G. McKittrick, D. Beede, B. Khan, and M. Doms. 2011. STEM: Good jobs now and for the future (Issue Brief \#03-11). Washington, DC: U.S. Department of Commerce, Economics and Statistics Administration.
[16] O. Lüdtke, A. Robitzsch, U. Trautwein, and M. Kunter. 2009. Assessing the impact of learning environments: How to use student ratings of classroom or school characteristics in multilevel modeling. Contemporary Educational Psychology, 34(2), 120-131.

[17] J. J. McConnell. 1996. Active learning and its use in computer science. ACM SIGCSE Bulletin, 28(SI), 52-54.

[18] L. K. Muthén and B. O. Muthén. 1998-2017). Mplus user's guide. 8th ed. Muthén \& Muthén, Los Angeles, CA.

[19] M. S. Peteranetz, A. E. Flanigan, D. F. Shell, and L.-K. Soh. 2018. Future-oriented motivation and retention in computer science. In SIGCSE '18: 49th ACM Technical Symposium on Computing Science Education, Feb. 21-24, 2018, Baltimore, MD, USA. ACM, NY, NY, USA, 6 pages. https://doi.org/10.1145/3159450.3159513

[20] K. G. Nelson, D. F. Shell, J. Husman, E. J. Fishman, and L.-K. Soh. 2015. Motivational and self-regulated learning profiles of students taking a foundational engineering course. Fournal of Engineering Education, 104(1), 74-100.

[21] L. Ott, B. Bettin, and L. Ureel. 2018, July. The impact of placement in introductory computer science courses on student persistence in a computing major. In Proceedings of the 23rd Annual ACM Conference on Innovation and Technology in Computer Science Education (pp. 296-301). ACM.

[22] M. Overmars. 2004. Teaching Computer Science through Game Design, Computer, 37(4), 81-83

[23] M. S. Peteranetz, and L.-K. Soh. 2019. Predicting retention in undergraduate computer science courses: Perceived instrumentality and attendance. Presented at the annual meeting of the American Educational Research Association, Toronto, Ontario, Canada.

[24] L. Porter, M. Guzdial, C. McDowell, and B. Simon. 2013. Success in introductory programming: What works?. Communications of the ACM, 56(8), 34-36.

[25] K. Pulvers, and G. M. Diekhoff. 1999. The relationship between academic dishonesty and college classroom environments. Research in Higher Education, 40(4), 487-498.

[26] E. Seymour and N. M. Hewitt. 1997. Talking about leaving: Why undergraduates leave the sciences. Westview Press, Boulder, $\mathrm{CO}$

[27] D. F. Shell, M. P. Hazley, L.-K. Soh, E. Ingraham, and S. Ramsay. 2013, October. Associations of students' creativity, motivation, and self-regulation with learning and achievement in college computer science courses. In Proceedings of the 2013 IEEE Frontiers in Education Conference (FIE) (pp. 1637-1643). IEEE

[28] J. P. Somervell. 2006, June. Pair Programming: Not for Everyone?. In Proceedings of the International Conference on Frontiers in Education: Computer Science and Computer Engineering, (pp. 303-307).

[29] K. Umapathy and A. D. Ritzhaupt. 2017. A meta-analysis of pair-programming in computer programming courses: Implications for educational practice. ACM Transactions on Computing Education (TOCE), 17(4), 16.

[30] J. Watkins and E. Mazur. 2013. Retaining students in science, technology, engineering, and mathematics (STEM) majors. Journal of College Science Teaching, 42(5), 36-41.

[31] Z. S. Wilson, et al. 2011. Hierarchical mentoring: A transformative strategy for improving diversity and retention in undergraduate STEM disciplines. Fournal of Science Education and Technology, 21(1), 148-156.

[32] M. R. Young. 2005. The motivational effects of the classroom environment in facilitating self-regulated learning. Journal of Marketing Education, 27(1), 2540. 